\title{
Local Freeway Ramp Metering using Self-Adjusted Fuzzy Controller
}

\author{
Hongyan GAO, Rundong RAN
}

\begin{abstract}
A self-adjusted fuzzy local ramp metering strategy is proposed to keep the mainline traffic state and the on-ramp queue length at reasonable levels. The fuzzy ramp metering strategy (FRMS) takes the following variables as inputs: error between desired density and measured density, change-in-error and on-ramp queue length. On-ramp metering flow is decided by these variables. It is difficult to construct fuzzy rules for a three-dimension inputs fuzzy controller based on expert knowledge, so the proposed FRMS generates fuzzy control rules by an analytic expression with correction factors. The correction factors reflect the weights upon linguistic variables of inputs and can be regulated according to actual traffic state of mainline and on-ramp. The proposed FRMS not only simplifies the process of rules definition for a multi-dimension fuzzy controller, but also has function of self-adjusted control rules. To examine the proposed FRMS, a freeway stretch in Los Angeles is simulated with distributed models. The proposed FRMS is also compared with an existing T-S FRMS and PI-ALINEA in the simulation experiments which cover different on-ramp inflow scenarios. Simulation results show the proposed FRMS provides improved adaptation to various scenarios and superiority in striking a balance between the mainline and on-ramp performances.
\end{abstract}

Keywords: balance; correction factors; fuzzy controller; mainline density; on-ramp queue length; ramp metering; self-adjusted rules

\section{INTRODUCTION}

Ramp metering, based on regulating the entering traffic to the freeway, has been recognized as an effective and economic way to relieve freeway congestion $[1,2]$. Over the years, a number of ramp metering strategies including local responsive and coordinated ramp metering have been exploited. Local ramp metering strategies such as demand-capacity, occupancy control, and ALINEA [3] regulate on-ramp flow into mainline to avoid congestion on mainline. Different from demand-capacity and occupancy control, ALINEA is a closed-loop local metering algorithm with multiple successful field applications [4]. Recently, Wang and Papageorgiou suggested PI-ALINEA which is a Proportional-Integral extension of ALINEA [5]. In addition, the iterative learning control (ILC) based ramp metering methods have been proposed to keep mainline density at a desired level [6-9]. Hou et al. [6] exploited the pure ILC-based ramp metering approach. In [7], ILC-based ramp metering approach combined the learning mechanism with an error feedback. In [8], the modified ILC add-on to ALINEA has been analysed. Chi et al. [9] presented a new queue length information fusion based iterative learning control approach for freeway traffic ramp metering. In addition, some advanced control strategies such as fuzzy logic control and neural networks control [10] were developed in local ramp metering. Coordinated ramp metering aims at optimizing traffic flow over a section of the freeway by metering all on-ramps within the section. Typical coordinated ramp metering strategies include METALINE, FLOW, Zone algorithm, Helper and SWARM. Some field operational tests [11] and simulation $[12,13]$ evaluated the performance of the local and coordinated ramp metering strategies and concluded that there were no significant differences between them in many cases. Winyoopadit [14] proved that ALINEA was superior to FLOW and stratified Zone under moderatehigh-demand. In contrast to local ramp metering, coordinated approach is very complex and expensive to implement.

Affected by stochastic factors such as incidents, bad weather, drivers' behaviours, inaccurate measures and special events, traffic flow has the characteristics of uncertainty, complexity and randomness. Therefore, it is hardly available to obtain accurate mathematical model for traffic flow, which makes analytic control algorithm ineffective in practice. Fuzzy logic control (FLC) appears to be more suitable for ramp metering than analytic control algorithm because it can utilize imprecise or ambiguous information and regulate traffic flow in a way similar to human reasoning. Fuzzy logic control handles imprecise information with linguistic description and uses rulesbased logic to incorporate human knowledge. These capabilities allow fuzzy logic control to tune the algorithm easily to accommodate fluctuation of traffic flow. Since 1990, fuzzy logic control has been successfully implemented in ramp metering system. A research group designed a fuzzy controller with six inputs and three outputs for freeway ramp metering and proved its efficiency in reducing congestion [15]. In Holland, a fuzzybased ramp metering algorithm was tested on an 11-km freeway section and produced 35 percent faster travel times than two other methods [16]. A group from University of Washington implemented fuzzy logic control in the greater Seattle area and compared its performance with the Local and Bottleneck ramp metering algorithms [17]. Overall, the effect of fuzzy control appeared superior to the other algorithms. A Takagi-Sugeno based fuzzy ramp metering strategy (T-S FRMS) similar to ALINEA was studied to balance traffic conditions on the mainstream and the onramp [18]. Different from ALINEA, the desired mainstream density in T-S FRMS is not a constant but decided by the FLC-based algorithm according to actual traffic state. Chiang and Wang [19] integrated a fuzzy logic and a decision-making model in ramp metering system and tested its advantage over pre-timed ramp metering. Chen et al. [20] proposed ramp metering based on fuzzy neural network for a junction and demonstrated its comparative efficiency. Pham et al. [21] combined fuzzy signal and ramp metering at a diamond interchange to improve traffic condition on surface streets and motorway. Ghodset et al. [22] integrated genetic-fuzzy ramp metering and variable speed limit control to solve freeway congestion. Recently, fuzzy ramp metering methods with optimization algorithms were exploited and showed better performance than FLC without optimization or ALINEA [23-25]. 
Most existing ramp metering methods are efficient in maintaining proper traffic state of freeway mainline. Onramp is a connection, which allows vehicles to enter freeway mainline. The storage space of on-ramp is limited. If ramp metering strategy is applied to smooth the mainline traffic state without considering the limited storage space of on-ramp, it is likely to cause on-ramp spillover and affect the attached urban network. In order to striking a balance between the mainline and on-ramp performances, a fuzzy-based ramp metering strategy is proposed in this paper. In general, fuzzy logic control involves three main steps: fuzzification, fuzzy inference and defuzzification. Fuzzification transforms inputs into linguistic variables. Fuzzy inference implements the control heuristics based on rules base. Defuzzification converts linguistic variables to quantitative state. As the heart of fuzzy logic controller, the rules base consists of a set of if-then statements, which derive from expert opinions and operator experience. A fuzzy logic control for ramp metering usually comprises multi-dimension inputs because many types of information such as traffic state of mainline and on-ramp should be considered. Considering the traffic state of mainline and on-ramp, the proposed fuzzy ramp metering strategy takes three variables as inputs: error between desired density and measured density, change-in-error and on-ramp queue length. Fuzzy control rules expand exponentially with the dimension of inputs and the number of fuzzy sets for input and output. For example, for a three-dimension inputs and one-dimension output controller, if we choose five fuzzy sets for each input and output, the complete rule base consists of $5^{3}=125$ rules. Obviously, it is difficult to establish rules base only depending on the human experience for a multi-dimension inputs ramp metering system. In addition, once the rules base and fuzzy inference are determined, the adaptability of the fuzzy controller becomes poor. Long and Wang [26] proposed an approach to construct fuzzy control rules by an analytic expression with a correction factor. This method can not only overcome the difficulty of determining rules by experience for a multi-dimension inputs controller, but also guarantee the completeness and on-line regulation of fuzzy rules. Based on the approach proposed by Long and Wang, the proposed FRMS generates fuzzy control rules by an analytic expression with correction factors. The proposed FRMS has ability to adjust fuzzy rules in process of on-line control according to traffic state of mainline and on-ramp. Furthermore, it offers a trade-off solution between mainline density control and on-ramp queue control.

\section{DISTRIBUTED MODELS DESCRIPTION}

\subsection{Macroscopic Traffic Model for Freeway}

Suppose a freeway is divided into segments (indicated by the index $i$ ) with length $\Delta x_{i}$. Each segment has no more than one on-ramp and one off-ramp. The study uses the following macroscopic traffic model [27] to describe the freeway:

$$
\begin{aligned}
& \rho_{i}(k+1)=\rho_{i}(k)+\frac{T}{\lambda_{i} \Delta x_{i}}\left[q_{i-1}(k)-q_{i}(k)+u_{i}(k)-s_{i}(k)\right](1) \\
& q_{i}(k)=\lambda_{i} \cdot \rho_{i}(k) \cdot v_{i}(k)
\end{aligned}
$$

$$
\begin{aligned}
& v_{i}(k+1)=v_{i}(k)+\frac{T}{\tau}\left[V\left(\rho_{i}(k)\right)-v_{i}(k)\right]+ \\
& \frac{T}{\Delta x_{i}} v_{i}(k)\left[v_{i-1}(k)-v_{i}(k)\right]-\frac{\mu T}{\tau \Delta x_{i}} \frac{\rho_{i+1}(k)-\rho_{i}(k)}{\rho_{i}(k)+\theta}
\end{aligned}
$$

where $T$ is the sample time interval, $\lambda_{i}$ is number of lanes in the segment $i, k$ indicates the time step $t=k T(k=1,2, \ldots)$, $\rho_{i}(k)$ is traffic density in segment $i$ at time step $k T, q_{i}(k)$ is traffic flow leaving segment $i$ entering next segment at time step $k T, u_{i}(k)$ is on-ramp metering flow entering segment $i$ at time step $k T$ if segment $i$ has one on-ramp, else $u_{i}(k)=0 . s_{i}(k)$ is off-ramp flow leaving segment $i$ at time step $k T$ if segment $i$ has one off-ramp, else $s_{i}(k)=0 . \tau, \mu$ and $\theta$ are constant parameters which reflect freeway characteristics. Eq. (1) is conservation equation, Eq. (2) is the dynamic flow equation, and Eq. (3) is dynamic speed equation.

In Eq. (3), $V\left[\rho_{i}(k)\right]$ represents the speed-density relationship. Various mathematical models have been developed to describe the speed-density relationship, such as linear model [28], logarithmic model [29], exponential model [30-32] and two-regime model [33]. Therefore, Eq. (1), Eq. (2) and Eq. (3) make up the macroscopic traffic model for freeway. Obviously, the macroscopic traffic model is a nonlinear equation which describes traffic flow dynamic process given initial density.

\subsection{On-ramp Queue Model and Constraint Conditions}

For a segment $i$ with on-ramp, let $u_{i}(k)$ be on-ramp metering flow entering segment $i$ at time step $k T, r_{i}(k)$ be the inflow entering the ramp $i, l_{i}(k)$ be on-ramp queue length of ramp $i$. Due to the conversation law, on-ramp queue dynamics is modeled as follows:

$l_{i}(k+1)=l_{i}(k)+T\left[r_{i}(k)-u_{i}(k)\right]$

Since the on-ramp queue length cannot be negative and cannot exceed the maximum ramp length $l_{\max }$, Eq. (1) satisfies:

$0 \leq l_{i}(k)+T\left[r_{i}(k)-u_{i}(k)\right] \leq l_{\max }$

Besides, maximum ramp metering flow $u_{\max }$ and minimum ramp metering flow $u_{\text {min }}$ give the constraint conditions for ramp metering flow $u_{i}(k)$ :

$$
\begin{aligned}
& u_{i}(k) \geq \max \left\{r_{i}(k)+\left[l_{i}(k)-l_{\max }\right] / T, u_{\min }\right\} \\
& u_{i}(k) \leq \min \left\{r_{i}(k)+l_{i}(k) / T, u_{\max }\right\}
\end{aligned}
$$

\section{PRINCIPLE OF FLC WITH CORRECTION FACTOR}

For a fuzzy controller with two-dimension inputs and one-dimension output, suppose $E$ and $E C$ are linguistic variables of the inputs with universe of discourse $X$ and $Y$ respectively, and $U$ is linguistic variable of the output with universe of discourse $Z$. The fuzzy set of linguistic variables $E, E C$ and $U$ are denoted as follows: 


$$
\begin{array}{ll}
E=\left\{E_{i}\right\} \in F(X), & i=1,2, \ldots, n_{1} \\
E C=\left\{E C_{j}\right\} \in F(Y), & j=1,2, \ldots, n_{2} \\
U=\left\{U_{k}\right\} \in F(Z), & k=1,2, \ldots, n_{3}
\end{array}
$$

where $F(X)$ is the fuzzy power set of $X[34], n_{1}, n_{2}$ and $n_{3}$ are the numbers of the fuzzy subsets of $E, E C$ and $U$ respectively.

The fuzzy rules are usually derived from human knowledge or experience and expressed in IF-THEN form as follows:

If $E$ is $E_{i}$ and $E C$ is $E C_{j}$, Then $U$ is $U_{k}$.

Long and Wang [21] used an analytic expression to describe the above rules:

$$
U=\langle\zeta E+(1-\zeta) E C\rangle, \quad \zeta \in(0,1)
$$

where $\langle\cdot>$ is round function which rounds a number to the nearest integer, and $\zeta$ is a correction factor. $\zeta$ and $(1-\zeta)$ represent the weighted values of $E$ and $E C$ respectively. So the fuzzy rules change with different value of $\zeta$.

\section{DESIGN OF FUZZY RAMP METERING \\ 4.1 Input and Output Variables Selection}

The main task of the proposed fuzzy ramp metering is to maintain traffic downstream of on-ramp at a reasonable level. In addition, it also takes on-ramp queue spillover into account. So the error between the desired density and measured downstream density by detectors, change-inerror and on-ramp queue length are selected as input variables. The output of fuzzy controller is the deviation of ramp metering flow. Therefore, the fuzzy ramp metering is a local-feedback controller with three-dimension inputs and a single output. Denote the error, change-in-error, onramp queue length and deviation of ramp metering flow at time instant $k T$ as $e(k), e c(k), l(k)$ and $\Delta u(k)$ respectively. Error $e(k)$ is defined by $e(k)=\rho_{\mathrm{d}}-\rho(k)$, where $\rho_{\mathrm{d}}$ is the desired value for the downstream density and $\rho(k)$ is measured density at time instant $k T$. Change-in-error $e c(k)$ is the difference between measured values for density in two consecutive intervals, that is $e c(k)=[e(k)-e(k-1)] / T$. Change-in-error reflects the trend of error change.

\subsection{Fuzzification}

Assume that $E, E C$ and $L$ are linguistic variables of inputs $e(k), e c(k)$ and $l(k), U$ is linguistic variable of output $\Delta u(k) . E, E C$ and $U$ all comprise five fuzzy subsets: negative big(NB), negative small(NS), zero(ZO), positive small(PS), positive big(PB). The normalized domain for these linguistic variables $E, E C$ and $U$ are $[-2,2],[-2,2]$ and $[-2,2]$. Five fuzzy subsets, very long(VL), long(L), middle(M), short(S) and very short(VS) are defined for onramp queue length $L$ with normalized domain [0,2].

Suppose that the actual physical domain of error, change-in-error, on-ramp queue length and deviation of ramp metering flow are $\left[-e_{1}, e_{1}\right],\left[-e c_{1}, e c_{1}\right],\left[0, l_{\max }\right]$ and $\left[-\Delta u_{1}, \Delta u_{1}\right]$ respectively. Scaling factors $K_{e}, K_{e c}$, and $K_{l}$ transform the physical domain $\left[-e_{1}, e_{1}\right],\left[-e c_{1}, e c_{1}\right],\left[0, l_{\max }\right]$ into the normalized domain $[-2,2],[-2,2],[0,2]$. On the contrary, scaling factor $K_{u}$ transforms the normalized domain $[-2,2]$ into the actual physical domain of output [$\left.\Delta u_{1}, \Delta u_{1}\right]$. These scaling factors are calculated by the following equation.

$$
K_{e}=2 / e_{1}, K_{e c}=2 / e c_{1}, K_{l}=2 / l_{\max }, K_{u}=\Delta u_{1} / 2
$$

So the measured value $e(k), e c(k), l(k)$ can be mapped into the normalized domain using appropriate scaling factors as follows.

$$
E=K_{e} \cdot e(k), \quad E C=K_{e c} \cdot e c(k), L=K_{l} \cdot l(k)
$$
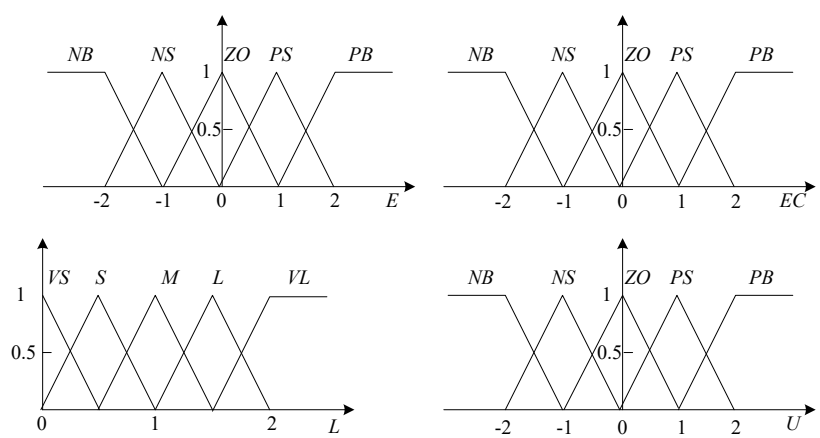

Figure 1 Membership function of $E, E C, L$ and $U$

The membership function for normalized inputs and output are described by triangular and trapezoidal function, as shown in Fig. 1. According to the membership function, the normalized input and output variables are fuzzified to the corresponding fuzzy subset.

\subsection{Control Rules and Inference}

Based on Eq. (8), the fuzzy control rules can be expressed by an analytic formula as follows

$U=\langle\alpha E+\gamma E C+\beta L\rangle$

where $\alpha, \gamma$ and $\beta$ are correction factors which represent the weights of $E, E C$ and $L$. Correction factors $\alpha, \gamma$ and $\beta$ satisfy the following condition:

$\alpha+\gamma+\beta=1 \quad$ s.t. $\alpha, \gamma, \beta \in[0,1]$

So Eq. (11) can be written as:

$U=\langle\alpha E+(1-\alpha-\beta) E C+\beta L\rangle$

It can be seen from Eq. (13), if the correction factors $\alpha$ and $\beta$ are set constants in advance, the fuzzy control rules cannot be changed any longer during on-line control. A good fuzzy controller for ramp metering should have the performance of on-line adjusting fuzzy control rules because of fluctuation from upstream and on-ramp demand. So the correction factors are defined in fuzzy form instead of the constants in our study. The correction factors in fuzzy form can be regulated according to different error, change-in-error and queue length in real control system. In this way, the fuzzy control rules can be changed flexibly with different value of the correction factors. 
Firstly, correction factor $\beta$ is determined by queue length $L$ and error $E$ according to fuzzyfication, fuzzy inference and defuzzyniess. Five linguistic expressions "zero(ZO)", "very small(VS)", "small(S)", "big(B)" and "very big(VB)" describe correction factor $\beta$ in fuzzy form denoted as $B T$. The membership function of $B T$ is specified in Fig. 2.

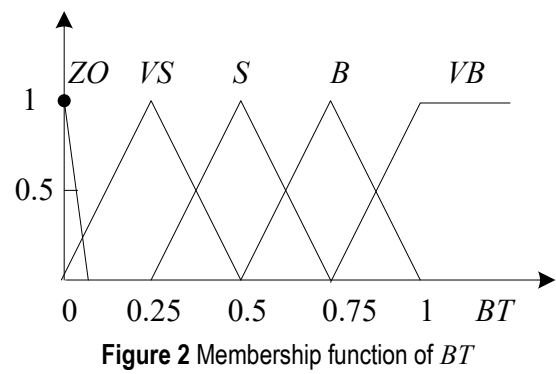

When queue length is short, the main task is to ensure that traffic density does not exceed the critical density and to make system stabilize at the desired density as much as possible, thus $\beta$ should be smaller. When queue length is long, the main task is to keep the queue length below an allowable value as much as possible, thus $\beta$ should be bigger. Based on the above thought, the fuzzy control rules about queue length $L$ and error $E$ with correction factor $B T$ are described as shown in Tab. 1.

Table 1 Fuzzy control rules about queue length $L$ and error $E$ with correction

\begin{tabular}{|c|c|c|c|c|c|c|}
\multicolumn{8}{|c|}{ factor $B T$} \\
\cline { 3 - 8 } Correction factor $(B T)$ & \multicolumn{5}{|c|}{ Error $(E)$} \\
\hline \multirow{4}{*}{ Queue length $(L)$} & VS & ZO & ZO & ZO & ZO & ZO \\
\cline { 2 - 8 } & $\mathrm{S}$ & ZO & VS & VS & VS & VS \\
\cline { 2 - 8 } & M & ZO & S & S & S & B \\
\cline { 2 - 8 } & L & VS & B & B & VB & VB \\
\cline { 2 - 8 } & VL & S & B & VB & VB & VB \\
\hline
\end{tabular}

The above rules can be denoted as fuzzy relationship $R$ between $L, E$ and $B T$. Fuzzy output variable $B T$ is obtained based on Mamdani inference [35]:

$$
B T=(L \times E) \circ R
$$

Then the fuzzy output $B T$ is defuzzified by the center of gravity (COG) function into a crisp value $\beta$. Because the correction factor $\beta$ for ramp queue length $L$ is determined by $L$ and $E$, the correction factor $\alpha$ for error $E$ should contain weight $(1-\beta)$. In addition, another weight for the correction factor $\alpha$ depends on error $E$ and change-in-error $E C$. When error is big, the weighted value of error is assigned bigger than that of change-in-error in order to eliminate the error as fast as possible. When error is small, the weighted value of error is assigned smaller than that of change-in-error in order to make system stability as fast as possible and avoid a larger overshoot [36]. Combining the weight $(1-\beta)$ and another weight decided by $E$ and $E C$, correction factor $\alpha$ is expressed as follows:

$\alpha=\frac{|E|}{|E|+|E C|}(1-\beta)$
Eq. (14) and Eq. (15) show that correction factors $\alpha$ and $\beta$ can be self-regulated according to the change of error, change-in-error and queue length. So the fuzzy control rules can be revised on-line with the self-regulated factors $\alpha$ and $\beta$.

Finally, the output $U$ from Eq. (13) is transformed into the actual physical domain for deviation of ramp metering flow by scaling factor $K_{u}$ :

$\Delta u(k)=K_{u} \cdot U$

The ramp metering flow $u(k)$ at time instant $k T$ is calculated as follows:

$u(k)=u(k-1)+\Delta u(k)$

From above analysis, the proposed fuzzy ramp metering system is constructed as shown in Fig. 3. In Fig. $3, q_{u}(k)$ and $r_{i n}(k)$ are upstream demand and on-ramp inflow respectively at time instant $k T$.

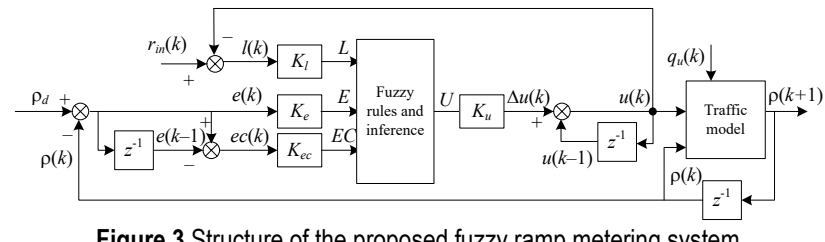

Figure 3 Structure of the proposed fuzzy ramp metering system

\section{SIMULATION EXPERIMENT \\ 5.1 Experiment setup}

In order to evaluate the proposed strategy, a freeway stretch in Los Angeles is considered as study site (see Fig. 4). The freeway stretch is divided into four segments with length $\Delta x_{i}, \Delta x_{1}=1094 \mathrm{~m}, \Delta x_{2}=885 \mathrm{~m}, \Delta x_{3}=885 \mathrm{~m}, \Delta x_{4}$ $=1046 \mathrm{~m}$. The mainline has three lanes with capacity of about $5200 \mathrm{veh} / \mathrm{h}$. The on-ramp R2 with one lane is about $300 \mathrm{~m}$ from link entrance to stop line.

In order to depict the real traffic state, measured upstream demand $q_{u}$ from detector 1, on-ramp demand $r_{1}$ from detector 3 and on-ramp demand $r_{2}$ from detector 4 with time intervals of 5 minutes are employed as inputs of the experiment. The measured data are provided by California Department of Transportation Performance Measurement System (PeMs). The experiment simulates traffic state from 7:00 am to 9:00 am on November 12, 2014. The simulation time step and control sample time are $10 \mathrm{~s}$ and $30 \mathrm{~s}$. Fig. 5 shows the aggregate traffic demand of upstream $\left(q_{\mathrm{u}}\right)$ and two on-ramps which are converted to hourly flow. The total demand entering the second segment does not exceed its capacity in peak hour, so no-control measure is applied to on-ramp R1. The speed measured by detector 2 shows recurrent congestion occurred on the fourth segment in peak hour because the total demand entering the fourth segment exceeds its capacity. The ramp metering strategies are only applied to on-ramp R2 to regulating the entering traffic to the fourth segment. To reflect different traffic state of the downstream and evaluate the performance of different ramp metering strategy, three simulation scenarios cover different onramp (R2) inflow: 
Scenario 1: on-ramp inflow $r_{i n}$ is the measured onramp demand $r_{2}$, that is $r_{i n}=r_{2}$

Scenario 2: on-ramp inflow is an increase of $10 \%$ of the measured on-ramp demand $r_{2}$, that is $r_{\text {in }}=1.1 r_{2}$

Scenario 3: on-ramp inflow is an increase of $20 \%$ of the measured on-ramp demand $r_{2}$, that is $r_{i n}=1.2 r_{2}$

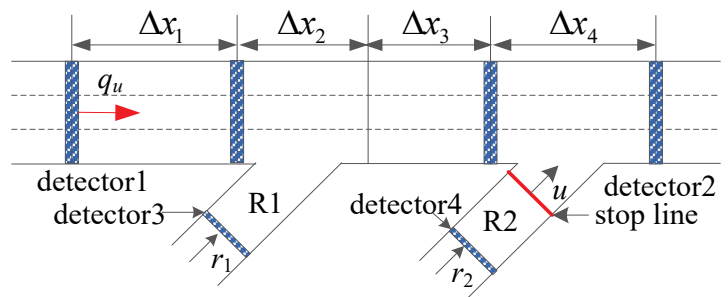

Figure 4 Study site

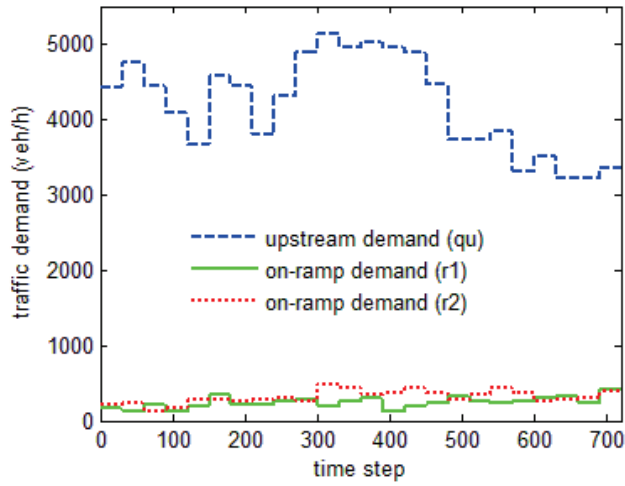

Figure 5 The measured traffic demand of upstream and on-ramp by detectors

The simulation experiments follow the macroscopic traffic model and on-ramp queue model discussed in section 2 . The study adopts different mathematical models to fitting the measured data and chooses the most appropriate model to describe the speed-density relationship.

$V\left[\rho_{i}(k)\right]=v_{f}\left[1-\left(\rho_{i}(k) / \rho_{j a m}\right)^{\delta}\right]^{m}$

where $v_{f}$ is free-flow speed, $\rho_{\text {jam }}$ is jam density, $\delta$ and $m$ are parameters. According to the measured data, the study sets $v_{f}=112 \mathrm{~km} / \mathrm{h}, \rho_{\text {jam }}=58 \mathrm{veh} / \mathrm{km} / \mathrm{ln}, \delta=1.1$ and $m=1$. The above model shows that the capacity of the study segments is about $1733 \mathrm{veh} / \mathrm{ln}$ and the critical density $\rho_{c}$ corresponding to the segment capacity is about 30 $\mathrm{veh} / \mathrm{km} / \mathrm{ln}$.

\subsection{Evaluated Ramp Metering Strategies}

For each scenario, the proposed fuzzy ramp metering strategy is compared to another typical non-fuzzy ramp metering strategy known as PI-ALINEA[5] and to another existing T-S FRMS in work[18]. PI-ALINEA is described as:

$$
u(k)=u(k-1)-K_{P}[\rho(k)-\rho(k-1)]+K_{R}\left[\rho_{d}-\rho(k)\right]
$$

where $K_{P}$ and $K_{R}$ are gain factors for proportional term and integral term. In the study, $K_{P}=10$ and $K_{R}=40$ were found to lead to the best results.
The T-S FRMS used the following ramp metering strategy similar to ALINEA:

$$
u(k)=u(k-1)+K_{R}\left[\rho_{d}(k)-\rho(k)\right]
$$

where the desired density $\rho_{d}(k)$ is determined by the product of a predefined value $\rho_{r}$ and a quantity $c_{\rho}$, which is determined by FLC. The FLC takes density $\rho$, speed of mainstream flow $v$ and on-ramp queue length $l$ as fuzzy controller inputs. The fuzzy rules are described as follows:

IF $\rho$ is $W^{i}(\rho)$ AND $v$ is $W^{i}(v)$ AND $l$ is $W^{i}(l)$

THEN $c_{\rho}^{i}=\theta_{0}^{i}+\theta_{1}^{i} \rho+\theta_{2}^{i} v+\theta_{3}^{i} l$

where $\theta_{j}^{i}, j=0,1,2,3$ are parameters of the $i$ th fuzzy rule. The desired density $\rho_{d}(k)$ is eventually decided as follows:

$\rho_{d}(k)=\rho_{r} \frac{\sum_{i} c_{\rho}^{i} \mu^{i}}{\sum_{i} \mu^{i}}$

The work [18] used particle swarm optimization to tune parameters $\theta_{j}^{i}$ to obtain minimum cost function value. This paper uses the same fuzzy rules and cost function as the work [18].

\subsection{Simulation Parameters Setting}

The parameters used in the simulation model are listed as follows: initial density $\rho(0)=13 \mathrm{veh} / \mathrm{km} / \mathrm{ln}$ for all segments, maximum on-ramp queue length $l_{\max }=50$ veh according to on-ramp length, initial queue length $l(0)=0$, minimum ramp metering flow $u_{\min }=120 \mathrm{veh} / \mathrm{h}$, maximum ramp metering flow $u_{\max }=900 \mathrm{veh} / \mathrm{h}$. The desired density $\rho_{d}$ is set to $31 \mathrm{veh} / \mathrm{km} / \mathrm{ln}$ rather than $30 \mathrm{veh} / \mathrm{km} / \mathrm{ln}$, whereas the maximum flow is achieved through multiple simulations. The constant parameters in Eq. (3) are set as follows: $\tau=36 \mathrm{~s}, \mu=35 \mathrm{~km}^{2} / \mathrm{h}, \theta=13 \mathrm{veh} / \mathrm{km}$.

The parameters of the proposed FRMS are set as follows: the actual physical domain of error, change-inerror, on-ramp queue length and deviation of ramp metering flow are $[-10 \mathrm{veh} / \mathrm{km} / \mathrm{ln},+10 \mathrm{veh} / \mathrm{km} / \mathrm{ln}],[-3$ $\mathrm{veh} / \mathrm{km} / \mathrm{ln},+3 \mathrm{veh} / \mathrm{km} / \mathrm{ln}],[0,+50 \mathrm{veh}]$ and $[-180 \mathrm{veh} / \mathrm{h}$, $+180 \mathrm{veh} / \mathrm{h}$ ] respectively. According to Eq. (9), the scaling factors $K_{e}=2 / 10=0.2, K_{e c}=2 / 3, K_{l}=2 / 50=0.04$ and $K_{u}$ $=180 / 2=90$.

\subsection{Simulation Results}

The simulation results including downstream (the fourth segment) density, speed, on-ramp (R2) queue length in each scenario are shown in Fig. 6 - Fig. 8.

The performance of the three strategies is measured by total time spent (TTS) on the entire system, which consists of two components: the total travel time $\left(T T T_{i}, i=1,2,3,4\right)$ on each segment and total waiting time (TWT) on the onramp R2. TTS is defined as: 
$T T S=\lambda_{i} T \sum_{i=1}^{4} \sum_{k=1}^{720} \rho_{i}(k) \Delta x_{i}+T \sum_{k=1}^{720} l(k)$

where the first term is $T T T$ and the second term is $T W T$.
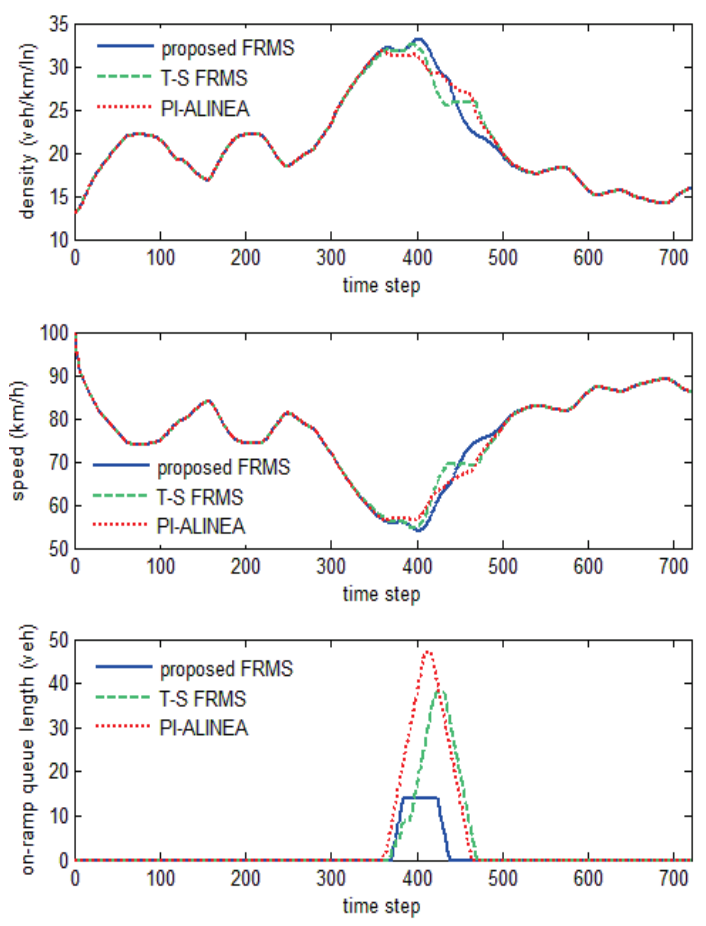

Figure 6 Simulation results in scenario 1
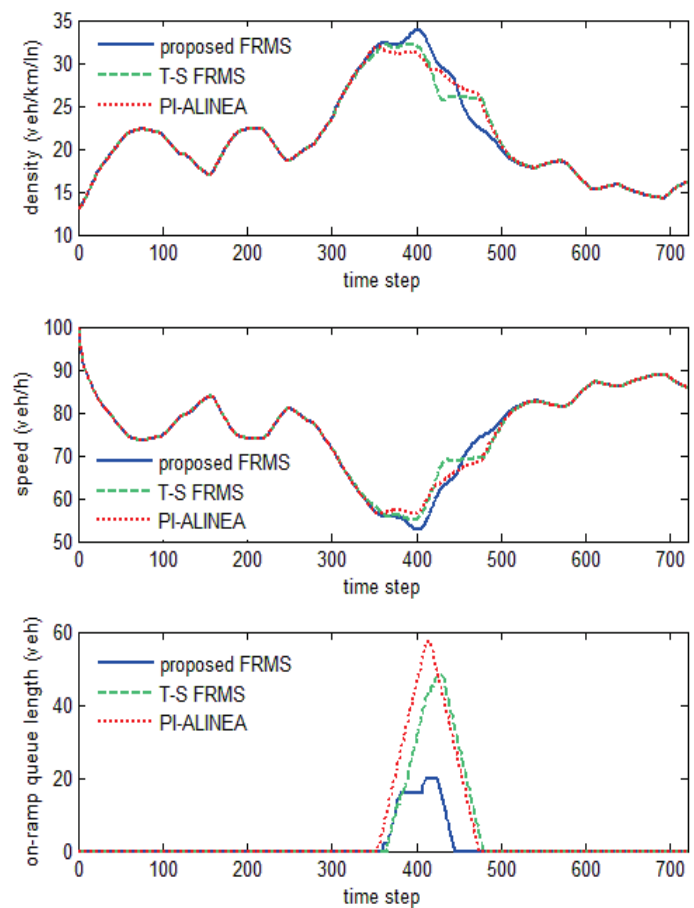

Figure 7 Simulation results in scenario 2

Tab. 2 compares total travel time (TTT), total waiting time $(T W T)$ and total time spent (TTS) for the three scenarios using the proposed FRMS, the T-S FRMS and PI-ALINEA. It can be seen from Tab. 2, the TTT on segment 1 and 2 controlled by different strategies are very close in all scenarios. In addition, Fig. 6-8 show that the simulation results of all evaluated strategies in all investigated scenarios are virtually similar over the first
300 step time and the last 180 step time of the simulation, so another performance index $T T S^{\prime}$ which reflects different control results of all evaluated strategies on segment 3 and 4 during step time 301 and 540 is also calculated as follows:

$$
T T S^{\prime}=\lambda_{i} T \sum_{i=3}^{4} \sum_{k=301}^{540} \rho_{i}(k) \Delta x_{i}+T \sum_{k=301}^{540} l(k)
$$
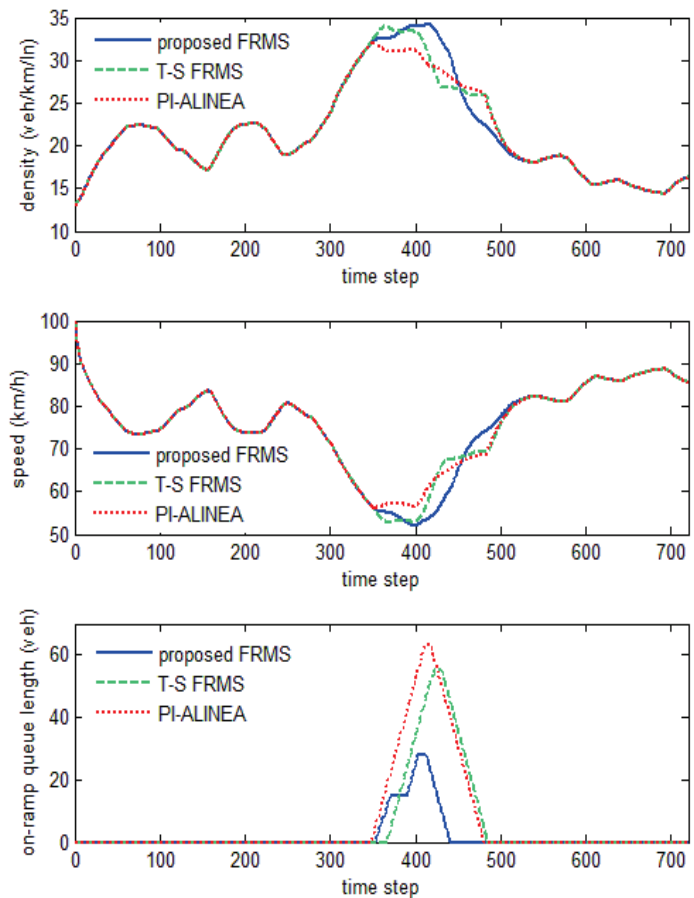

Figure 8 Simulation results in scenario 3

Table 2 Comparison results for $T T T, T W T, T T S$ and $T T S^{\prime}(\mathrm{veh} \cdot \mathrm{h})$

\begin{tabular}{|c|c|c|c|c|c|c|}
\hline \multirow[b]{2}{*}{$\begin{array}{l}\stackrel{.}{\Xi} \\
\bar{\Xi} \\
\mathscr{J} \\
\mathscr{n}\end{array}$} & \multirow[b]{2}{*}{ 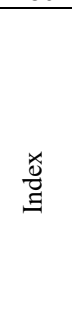 } & \multirow[b]{2}{*}{ 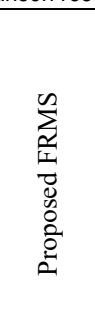 } & \multirow[b]{2}{*}{ 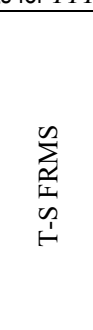 } & \multirow[b]{2}{*}{ 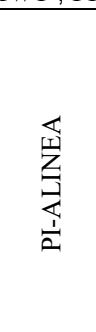 } & Change & Change \\
\hline & & & & & 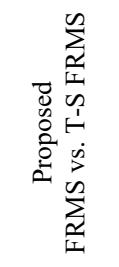 & 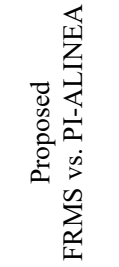 \\
\hline \multirow{8}{*}{1} & TTT1 & 113.63 & 113.63 & 113.62 & $0.00 \%$ & $0.01 \%$ \\
\hline & TTT2 & 98.37 & 98.34 & 98.34 & $0.03 \%$ & $0.03 \%$ \\
\hline & TTT3 & 101.11 & 101.08 & 101.06 & $0.03 \%$ & $0.05 \%$ \\
\hline & TTT4 & 131.73 & 131.69 & 131.59 & $0.03 \%$ & $0.11 \%$ \\
\hline & TTT & 444.83 & 444.74 & 444.61 & $0.02 \%$ & $0.05 \%$ \\
\hline & $T W T$ & 2.10 & 5.44 & 7.19 & $-61.39 \%$ & $-70.78 \%$ \\
\hline & TTS & 446.93 & 450.18 & 451.80 & $-0.72 \%$ & $-1.08 \%$ \\
\hline & $T T S^{\prime}$ & 97.53 & 100.70 & 102.83 & $-3.14 \%$ & $-5.15 \%$ \\
\hline \multirow{8}{*}{2} & TTT1 & 113.68 & 113.67 & 113.65 & $0.01 \%$ & $0.03 \%$ \\
\hline & TTT2 & 98.47 & 98.44 & 98.40 & $0.03 \%$ & $0.07 \%$ \\
\hline & TTT3 & 101.50 & 101.39 & 101.32 & $0.11 \%$ & $0.18 \%$ \\
\hline & TTT4 & 133.51 & 133.31 & 133.42 & $0.15 \%$ & $0.07 \%$ \\
\hline & TTT & 447.16 & 446.81 & 446.79 & $0.08 \%$ & $0.08 \%$ \\
\hline & $T W T$ & 3.13 & 8.28 & 9.98 & $-62.24 \%$ & $-68.64 \%$ \\
\hline & TTS & 450.29 & 455.09 & 456.76 & $-1.05 \%$ & $-1.42 \%$ \\
\hline & $T T S^{\prime}$ & 99.95 & 104.78 & 106.51 & $-4.61 \%$ & $-6.16 \%$ \\
\hline \multirow{8}{*}{3} & TTT1 & 113.74 & 113.74 & 113.67 & $0.00 \%$ & $0.06 \%$ \\
\hline & TTT2 & 98.61 & 98.60 & 98.46 & $0.01 \%$ & $0.15 \%$ \\
\hline & TTT3 & 102.03 & 101.93 & 101.54 & $0.10 \%$ & $0.48 \%$ \\
\hline & TTT4 & 135.82 & 135.46 & 134.65 & $0.27 \%$ & $0.87 \%$ \\
\hline & TTT & 450.19 & 449.73 & 448.32 & $0.10 \%$ & $0.42 \%$ \\
\hline & $T W T$ & 3.83 & 9.90 & 12.25 & $-61.29 \%$ & $-68.71 \%$ \\
\hline & TTS & 454.03 & 459.63 & 460.57 & $-1.22 \%$ & $-1.42 \%$ \\
\hline & $T T S^{\prime}$ & 102.68 & 108.30 & 109.43 & $-5.19 \%$ & $-6.17 \%$ \\
\hline
\end{tabular}


As an important evaluated index, the maximum onramp queue length using the three ramp metering strategies in all scenarios is shown in Tab. 3 .

Table 3 Maximum on-ramp queue length (veh)

\begin{tabular}{|c|c|c|c|}
\hline \multicolumn{4}{|c|}{ Table 3 Maximum on-ramp queue length (veh) } \\
\hline Scenario & Proposed FRMS & T-S FRMS & PI-ALINEA \\
\hline 1 & 14 & 41 & 47 \\
\hline 2 & 20 & 48 & 57 \\
\hline 3 & 28 & 55 & 63 \\
\hline
\end{tabular}

\section{ANALYSIS AND DISCUSSION}

Seen from Fig. 5, within the first 300 step time and the last 180 step time in all scenarios, the downstream traffic flow is less than the freeway capacity. Fig. 6-8 indicates that all evaluated strategies have the similar control results on downstream density, speed and on-ramp queue length during this period. Though the on-ramp metering flow of the three ramp metering strategies almost equals the onramp inflow, the downstream density in the three scenarios is always less than the desired density. Certainly, there is no on-ramp queue for the three ramp metering strategies. In this case, the three ramp metering strategies have no control effect since the downstream traffic flow is always below the freeway capacity.

As the upstream demand and on-ramp inflow increases, the downstream traffic flow gradually exceeds the freeway capacity. Fig. 6-8 indicate that the three ramp metering strategies have the different control results on downstream density, speed and on-ramp queue length during this period. In all scenarios, the downstream density and speed under PI-ALINEA have considerably similar profiles. PIALINEA maintains the downstream density around its critical value and keeps the downstream speed more than $56 \mathrm{~km} / \mathrm{h}$, a critical speed at which the flow is maximized, so no congestion appears on the mainline. For the proposed FRMS and T-S FRMS, the downstream density all exceed the critical value in different degree and slight congestion occurrs in all investigated scenarios. The T-S FRMS and the proposed FRMS show different profiles of the downstream density and speed in all scenarios. The maximum density under the T-S FRMS in scenario 1 and 2 is about $32.6 \mathrm{veh} / \mathrm{km} / \mathrm{ln}$, and increases to $34 \mathrm{veh} / \mathrm{km} / \mathrm{ln}$ in scenario 3. Acordingly, the speed under the T-S FRMS drops to around $54.6 \mathrm{~km} / \mathrm{h}$ in scenario 1 and 2, and 52.76 $\mathrm{km} / \mathrm{h}$ in scenario 3 . The downstream density controlled by the proposed FRMS takes on maximum deviation from the critical density in order to balance the on-ramp traffic state. The density deviation causes the decrease of speeds on the fourth segment. The transient and slight congestion at a threshold speed of $56 \mathrm{~km} / \mathrm{h}$ appeared under the proposed FRMS in all scenarios. In scenario 1, the slight congestion at a threshold speed of $56 \mathrm{~km} / \mathrm{h}$ lasts 45 time steps. In scenario 2, the downstream speed falls below $56 \mathrm{~km} / \mathrm{h}$ at time step 358 and increases back to $56 \mathrm{~km} / \mathrm{h}$ at time step 415. In scenario 3 , slight congestion on the fourth segment begins at time step 352 and ends at time step 427. The slight congestion dose not travel upstream and the slowest speed of the third segment is $57.36 \mathrm{~km} / \mathrm{h}$ in scenario 3 .

Seen from Fig. 6-8 and Tab.3, in all scenarios, PIALINEA has the longest on-ramp queue length and the proposed FRMS obtains the shortest on-ramp queue length among all evaluated strategies. The maximum on-ramp queue length controlled by PI-ALINEA in scenario 2 and
3 exceeds the allowed value and reaches 57 veh and 63 veh respectively. In contract, the proposed FRMS can restrict the maximum on-ramp queue length within its allowed value in all scenarios. Even if in scenario 3, the maximum on-ramp queue length controlled by the proposed FRMS is only 28 veh, which is far smaller than that of other strategies. In scenario 1 and 2, the T-S FRMS gives the efficient control on on-ramp queue length. Nevertheless, its control performance on on-ramp queue length starts to deteriorate in scenario 3 , and the maximum on-ramp queue length exceeds the allowed value.

The performance of the three on-ramp metering strategies is evaluated in terms of TTT, TWT, TTS and TTS'. It can be seen from Tab. 2, the TTT on segment 1 and 2 are quite similar in all scenarios for all strategies. In all scenarios, PI-ALINEA gains the most benefit on mainline performance, while makes vehicles suffer from the longest time on on-ramp. These results indicate that PI-ALINEA only lays emphasis on downstream density control without regard for the restriction of on-ramp queue length. Thus it produces the highest TTS on the entire system. On the contrary, in all scenarios, vehicles under the proposed FRMS control experience the lowest $T W T$ on on-ramp and the lowest TTS on the entire system. Compared with PIALINEA, the proposed FRMS increases TTT by 0.22 $\mathrm{veh} \cdot \mathrm{h}, 0.37 \mathrm{veh} \cdot \mathrm{h}$ and $1.87 \mathrm{veh} \cdot \mathrm{h}$ respectively in scenario 1-3, but it decreases $T W T$ by $5.09 \mathrm{veh} \cdot \mathrm{h}, 6.85 \mathrm{veh} \cdot \mathrm{h}$ and $8.42 \mathrm{veh} \cdot \mathrm{h}$ respectively. In scenario $1-3$, the proposed FRMS generates slightly higher TTT than the T-S FRMS does. However, the proposed FRMS decreases TWT by $61.39 \%, 62.24 \%$ and $61.29 \%$ respectively compared with the T-S FRMS. In scenarios 1 and 2, both the proposed FRMS and T-S FRMS can make a compromise between constraints on downstream density and on-ramp queue length. However, the T-S FRMS exceeds the queue length limit and reach 55veh in scenario 3. During time step 347 to 382 , density controlled by the T-S FRMS exceeds that of the proposed FRMS. But this trend dose not continue, and hence, larger on-ramp queue length produces from time step 383 to 483 . This results indicates that the balance action of the T-S FRMS is less efficient than that of the proposed FRMS as the traffic demand continues to increase. In terms of TTS, the performance of the proposed FRMS is superior to the T-S FRMS and PI-ALINEA. The performance index $T T S^{\prime}$ which reflects different control results of all evaluated strategies during congestion is also discussed. The proposed FRMS outperforms the T-S FRMS and PI-ALINEA by $3.14 \%$ and $5.15 \%$ decrease in scenario 1 , by $4.61 \%$ and $6.16 \%$ in scenario 2 , by $5.19 \%$ and $6.17 \%$ in scenario 3 . In all scenarios, the proposed FRMS shows its efficiency in managing on-ramp queue length within allowed value and providing moderate mainline density.

Overall, PI-ALINEA improves the mainline traffic state at the expense of on-ramp queue length. The T-S FRMS has ability to strike a balance between mainline and on-ramp traffic state when traffic demand is not very high. But its balance performance deteriorates as on-ramp demand increases. The T-S FRMS takes the error between the desired density and the measured density as its input. The desired density is optimized with fuzzy logic accordding to mainline density, speed and on-ramp queue length. The optimization process for the desired density is 
time-consuming. In addition, the frequent change of the desired density results in the stronger oscillations of control action. The proposed FRMS offers a trade-off solution between mainline density control and on-ramp queue control. The proposed FRMS takes the error, change-inerror, on-ramp queue length as the input variables of the fuzzy controller and weights these variables with fuzzy logic. The weights of these variablesare defined in fuzzy form instead of the constants and regulated according to the mainline desity and on-ramp queue length. So the fuzzy rules can be self-adjusted. Compared to the other strategies, the proposed FRMS has stronger adaptability to fluctuation of on-ramp inflow, which makes it highly robust.

\section{CONCLUSIONS}

This paper designed a self-adjusted fuzzy controller with three input variables for ramp metering. A simple method to generate rules by an analytic expression with correction factors is proposed. Correction factors are adjusted according to input variables so that fuzzy rules can be regulated flexibly in process of on-line control. This method can simplify the establishment of fuzzy rules and guarantee the rules completeness for a multi-dimension inputs controller. The proposed FRMS takes account of mainline traffic state and the on-ramp queue length. It balances both constraints on density and queue length in order to keep the mainline density and the on-ramp queue length at an acceptable value. The performance of the proposed FRMS, the T-S FRMS and PI-ALINEA is evaluated by simulation experiments covering three scenarios. The simulation results indicate that the proposed FRMS achieves a better balance between the main line and on-ramp performances and displays stronger robustness than the other strategies.

\section{REFERENCES}

[1] Papageorgiou, M. \& Kotsialos, A. (2002). Freeway ramp metering: an overview. IEEE Transactions on Intelligent Transportation Systems, 3(4), 271-281. https://doi.org/10.1109/TITS.2002.806803

[2] Hasan, M., Jha, M., \& Ben-Akiva, M. (2002). Evaluation of ramp control algorithms using microscopic traffic simulation. Transportation Research Part C, 10(3), 229-256. https://doi.org/10.1016/S0968-090X(02)00005-0

[3] Papageorgiou, M., Haj-Salem, H., \& Blosseville, J. (1991). ALINEA: A local feedback control law for on-ramp metering. Transportation Research Board, 1320, 58-64.

[4] Papageorgiou, M., Hadj-Salem, H., \& Midddelham, F. (1997). ALINEA local ramp metering: summary of field results. Transportation Research Board, 1603, 90-98. https://doi.org/10.3141/1603-12

[5] Wang, Y. B. \& Papageorgiou, M. (2014). Local ramp metering in the presence of a distant downstream bottleneck: theoretical analysis and simulation Study. IEEE Transactions on Intelligent Transportation Systems, 15, 2024-2039. https://doi.org/10.1109/TITS.2014.2307884

[6] Hou, Z. S., Xu, J. X., \& Zh, H. (2007). Freeway traffic control using iterative learning control-based ramp metering and speed signaling. IEEE Transactions on Vehicular Technology, 56(2), 466-477. https://doi.org/10.1109/TVT.2007.891431

[7] Hou, Z. S., Xu, J. X., \& Yan, J. W. (2008). An iterative learning approach for density control of freeway traffic flow via ramp metering. Transportation Research C: Emerging Technologies, 16(1), 71-97. https://doi.org/10.1016/j.trc.2007.06.007

[8] Hou, Z. S.; Yan, J. W.; Xu, J. X., et al. (2012). Modified Iterative-Learning-Control-Based Ramp Metering Strategies for Freeway Traffic Control With Iteration-Dependent Factors. IEEE Transactions on Intelligent Transportation Systems, 13(2), 606-618. https://doi.org/10.1109/TITS.2011.2174229

[9] Chi, R. H.; Li, M. Z.; Hou, Z. S., et al. (2014). Freeway traffic density and on-Ramp queue control via ILC approach. Mathematical Problems in Engineering, 1554, 1-8. https://doi.org/10.1155/2014/321934

[10] Zhang, H. M. \& Ritchie, S. G. (1997). Freeway ramp metering using artificial neural networks. Transportation Research Part C, 5(5), 273-286. https://doi.org/10.1016/S0968-090X(97)00019-3

[11] Neila, B., Habib, H., \& Jari, K. (2013) Isolated versus coordinated ramp metering: Field evaluation results of travel time reliability and traffic impact. Transportation Research Part C, 28(S1), 155-167.

[12] Masroor, H., Mithilesh, J., \& Moshe, B. (2002). Evaluation of ramp control algorithms usingmicroscopic traffic simulation. Transportation Research Part C, 10(3), 229-256. https://doi.org/10.1016/S0968-090X(02)00005-0

[13] Abdel-Aty, M. \& Gayah, V. (2010). Real-time crash risk reduction on freeways using coordinated and uncoordinated ramp metering approaches. Journal of Transportation Engineering, 136(5), 410-423. https://doi.org/10.1061/(ASCE)TE.1943-5436.0000100

[14] Winyoopadit, S. (2007). Development and comparative evaluation of ramp metering algorithms using Microscopic traffic simulation. Journalof Transport System Engineering and Information Technology, 7(5), 51-62. https://doi.org/10.1016/S1570-6672(07)60039-7

[15] Chen, L. L., May, A. D., \& Auslander, D. M. (1990). Freeway ramp control using fuzzy set theory for inexact reasoning. Transportation Research Part A, 24(1), 15-20. https://doi.org/10.1016/0191-2607(90)90067-G

[16] Taale, H., Slager, J., Rosloot, J. (1996). The assessment of ramp metering based on fuzzy logic. Proceedings of the Third Annual World Congress on Intelligent Transport Systems / Orlando, Florida, 101-108.

[17] Taylor, C., Meldrum, D., \& Jacobson, L. (1988). Fuzzy ramp metering: Design overview and simulation results. Transportation Research Record, 1634, 10-18. https://doi.org/10.3141/1634-02

[18] Xu, J. X.; Zhao, X. J.; \& Srinivasan, D. (2013). On optimal freeway local ramp metering using fuzzy logic control with particle swarm optimizations. IET Intelligent Transport System, 7(1), 95-104. https://doi.org/10.1049/iet-its.2012.0087

[19] Chiang, T. \& Wang, W. (2011). Highway on-ramp control using fuzzy decision-making. Journal of Vibration and Control, 17(2), 205-213. https://doi.org/10.1177/1077546309349854

[20] Chen, F., Jia, Y., Niu, Z. H., et al. (2011). Ramp metering research of junction based on fuzzy neural network model. Journal of Transport System Engineering and Information Technology, 11(1), 108-113. https://doi.org/10.1016/S1570-6672(10)60108-0

[21] Pham, V. C., Alam, F., Potgieter, J., et al. (2013). Integrated fuzzy signal and ramp metering at a diamond interchange. Journal of Advanced Transportation, 47(4), 413-434. https://doi.org/10.1002/atr.167

[22] Ghods, A. H., Kian, A. R., \& Tabibi, M. (2009). Adaptive ramp metering and variable speed limit control: A geneticfuzzy approach. IEEE Intelligent Transportation System Magazine, 1(1), 27-36. https://doi.org/10.1109/MITS.2009.932718 
[23] Yu, X. F. \& Alam, F. (2014). Genetic-fuzzy logic ramp metering control for rampmetering of motorway. Int. J. Computer Applications in Technology, 50(1), 30-44. https://doi.org/10.1504/IJCAT.2014.063906

[24] Zhu, P. (2008). An automatic tuning strategy for local fuzzy logic ramp metering algorithm using Particle Swarm Optimization. PhD thesis, Florida International University.

[25] Chai, G. \& Xie, Y. L. (2012). A genetic-fuzzy control application to ramp-mainstream joint coordination control of the freeway. Advanced Science Letters, 7(6), 369-374. https://doi.org/10.1166/asl.2012.2695

[26] Long, S. Z. \& Wang, P. Z. (1982). The self-regulation of fuzzy control rules. Fuzzy Mathematics, 2(3), 105-112.

[27] Payne, H. J. (1979). FREFLO: A macroscopic simulation model for freewaytraffic. Transportation Research Record, $722,68-77$

[28] Greenshields, B. D. (1933). The photographic method of studying traffic behaviors. Highway Research Board, 13(1), 382-399.

[29] Greenberg, H. (1959). An analysis of traffic flow. Operations Research, 7, 79-85. https://doi.org/10.1287/opre.7.1.79

[30] Drake, J. S., Schofer, J. L., \& May, A. D. (1967). A statistical analysis of speed density hypothesis. Highway Research Record, 154, 53-87.

[31] Drew, D. R. (1965). Deterministic aspects of freeway operations and control. Highway Research Record, 99, 4858.

[32] Pipes, L. A. (1967). Car-Following models and the fundamental diagram of road traffic. Transportation Research, 1, 21-29. https://doi.org/10.1016/0041-1647(67)90092-5

[33] Edie, L. C. (1961). Car-Following and steady-state theory for non-congested traffic. Operations Research, 9, 66-75. https://doi.org/10.1287/opre.9.1.66

[34] Wu, Z. Q., Wang, P. Z., \& Ten, H. H. (1992). A rules selfregulating fuzzy controller. Fuzzy Sets and system, 47(1), 1321. https://doi.org/10.1016/0165-0114(92)90055-9

[35] Mamdani, E. H. \& Assilian, S. (1975). An experiment in linguistic synthesis with a fuzzy logic controller. International Journal of Man-Machine Studies, 7(1), 1-13. https://doi.org/10.1016/S0020-7373(75)80002-2

[36]Liu, S. G., Wei, J. M., \& Zhu, Z. C. Fuzzy control technology. China Textile \& Apparel Press, China, 2001.

\section{Contact information:}

Hongyan GAO, Associate Professor, Dr

Corresponding author

College of Electrical Engineering and Automation,

Shandong University of Science and Technology,

266590 Qingdao, China

E-mail: gaohongyan0107@163.com

Rundong RAN, master graduate student

College of Electrical Engineering and Automation,

Shandong University of Science and Technology,

266590 Qingdao, China

E-mail: 422803513@qq.com 